\title{
ISOLATED ENTERIC SPLENIC LESION IN AN IMMUNOCOMPETENT HOST: AN INTERESTING CASE REPORT
}

\author{
ANJANA A ${ }^{1}$, PANKAJ SINGHAI ${ }^{2}$, RANJEETA ADHIKARY ${ }^{1}$, MALAVALLI V BHAVANA ${ }^{1}$, REDDI P YADAVALI ${ }^{3 *}$
}

${ }^{1}$ Department of Lab Medicine-Microbiology, Manipal Hospital, Bengaluru, Karnataka, India. ${ }^{2}$ Department of Internal Medicine, Manipal Hospital, Bengaluru, Karnataka, India. ${ }^{3}$ Department of Radiology-Microbiology, Manipal Hospital, Bengaluru, Karnataka, India. Email: yadavali@gmail.com

Received: 24 February 2021, Revised and Accepted: 30 March 2021

\section{ABSTRACT}

Splenic abscess is often an unrecognized complication of enteric fever. Diagnosis is difficult because of its rarity, insidious onset, and non-specific presentation. We report an interesting case of splenic lesion in an immunocompetent adolescent with no other comorbidities, who presented with history and clinical presentation more suggestive of tubercular etiology. However, culture from the CT-guided fine-needle aspirate grew Gram-negative bacilli, identified as Salmonella Typhi which was sensitive to ampicillin, cotrimoxazole, azithromycin, and ceftriaxone. He responded favorably with oral antibiotics without any further surgical intervention. High degree of clinical awareness with timely and appropriate microbiological evaluation helped into an early definitive diagnosis of enteric splenic abscess. This case highlights that in this era of emerging infections, we should not miss the atypical presentations of the endemic diseases. Safe and minimally invasive radiological intervention with good microbiological correlation is a successful spleen conserving treatment alternative to surgery in suitable patients of splenic abscess.

Keywords: Splenic abscess, Immunocompetent, Salmonella Typhi.

(C) 2021 The Authors. Published by Innovare Academic Sciences Pvt Ltd. This is an open access article under the CC BY license (http://creativecommons.org/ licenses/by/4.0/) DOI: http://dx.doi.org/10.22159/ajpcr.2021v14i5.41214. Journal homepage: https://innovareacademics.in/journals/index.php/ajpcr

\section{INTRODUCTION}

Splenic abscess is a rare entity among immunocompetent adults, with a reported incidence in autopsy series between $0.14 \%$ and $0.7 \%$ [1]. It has a diverse etiological profile which tends to change overtime. There is high risk of fatality if not diagnosed at an earlier stage. Diagnostic aspiration has a high yield in establishing the diagnosis and appropriate antibiotic therapy is the cornerstone of management [2]. We report an interesting case of splenic lesion clinically mimicking tubercular etiology, but was identified as enteric splenic abscess and successfully managed by noninterventional approach. The patient consent for the same was obtained.

\section{CASE REPORT}

An 18-year-old adolescent male, resident of West Bengal, presented to our outpatient department with a history of fever, malaise, and left side upper abdominal pain for a duration of 3 weeks. Fever was continuous, moderate grade which was more noticeable during evenings. The patient had already been on oral cefuroxime for the same, with little clinical improvement before presenting to our hospital. There was no history of predisposing factors such as hemoglobinopathies or HIV or trauma. On physical examination, the patient was afebrile with a blood pressure which was $90 / 60 \mathrm{mmHg}$. His abdominal examination revealed tenderness on the left hypochondrium with mild distention, while the remainder of his examination was unremarkable.

His blood parameters were not significant. Laboratory investigations revealed normocytic hypochromic anemia. His hematology profile was hemoglobin $9.0 \mathrm{~g} \%$, TLC $9800 / \mathrm{mm}^{3}$, and DLC: N 54, L 44, E 02, and M 00. Liver function test showed a mild elevation of transaminases (AST: 107; ALT: 162). Erythrocyte sedimentation rate (ESR) was $36 \mathrm{~mm} / \mathrm{h}$. Blood urea, creatinine, and electrolytes were within normal limits. Among the infectious disease markers, screen for malaria, dengue, and kala azar was negative. Widal test revealed insignificant titer of 1:80 for both TO and TH. Blood culture was sterile.

Multiphasic contrast-enhanced computer tomography (CECT) scan of abdomen and pelvis detected enlarged spleen with a span of $15 \mathrm{~cm}$.
A wedge-shaped, non-enhancing hypodense lesion of $4 \times 1.6 \mathrm{~cm}$ dimension was found in the anteroinferior part of the spleen. Crosssectional and longitudinal section view in multiphasic CECT scan of abdomen and pelvis (splenic lesion encircled) is shown in Figs. 1 and 2.

There is no wall enhancement or subcapsular collection. Mesenteric and retroperitoneal adenopathy without necrosis or calcification was also noted. An ultrasonography-guided aspiration of the lesion was performed and sent for pathological and infective analysis. Histopathological evaluation revealed numerous neutrophils with few histiocytes and degenerating inflammatory cells in a necrotic background. Xpert MTB/RIF assay for Mycobacterium tuberculosis complex detection was negative.

Aerobic culture of the aspirate yielded non-lactose fermenting colonies on MacConkey agar with typical biochemical reactions and Gram reactions. Growth on blood agar and MacConkey agar is shown in Fig. 3.

It was Gram-negative bacilli which was motile, alkaline/acid reaction without gas in triple sugar iron agar (TSI), citrate utilizing and positive reaction for methyl red reaction. Indole, urease, and Voges-Proskauer test were negative. Phenotypic identification and antibacterial susceptibility were performed using both conventional and automated VITEK-2 identification system (BioMérieux, France). Accordingly, it was identified as Salmonella enterica serotype Typhi, which was sensitive to ampicillin, cotrimoxazole, azithromycin, and ceftriaxone. Given the diagnosis of enteric splenic abscess, the patient was commenced on oral cotrimoxazole for a total duration of 4 weeks with vitamin supplements. He responded favorably without any further surgical intervention. On 6 months follow-up, he had clinically recovered and alleviated of symptoms.

\section{DISCUSSION}

Splenic abscess is a rare disease that occurs more often in immunocompromised patients [3]. The incidence of enteric splenic abscess has been reported to be between 0.29 and $2 \%$ [4]. It is often an unrecognized complication of enteric fever because of its 


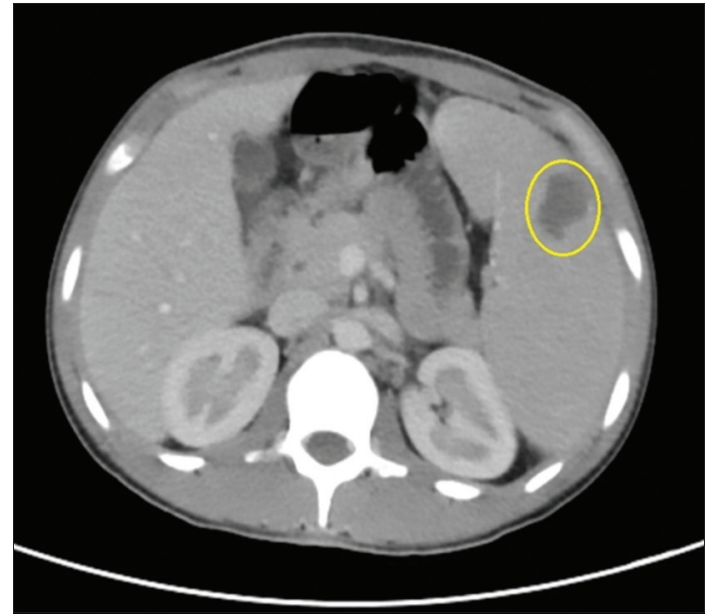

Fig. 1: Multiphasic CECT scan of abdomen and pelvis with the splenic lesion encircled (cross-section)

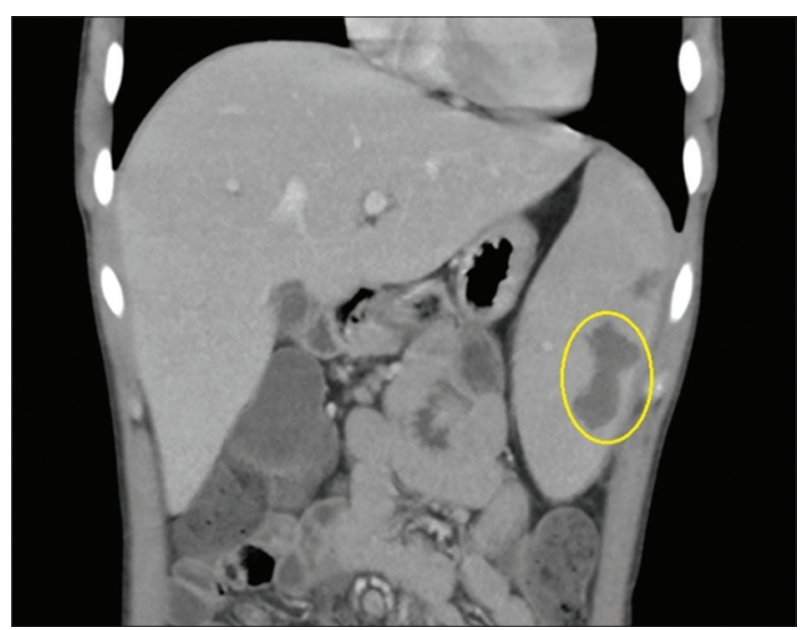

Fig. 2: Multiphasic CECT scan of abdomen and pelvis with the splenic lesion encircled (longitudinal section)

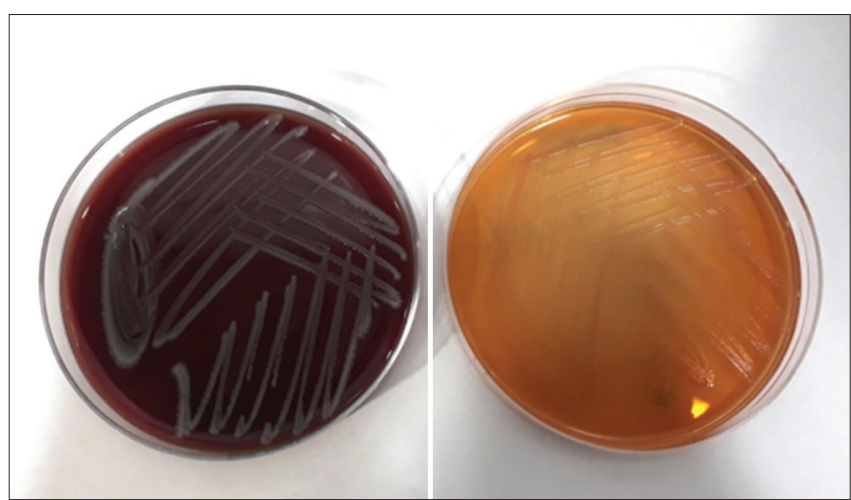

Fig. 3: Growth on blood agar and MacConkey agar

non-specific and polymorphic clinical presentation. Splenic abscess related to typhoid fever was most frequent in the pre-antibiotic era, nowadays, cause is AIDS, abdominal infections, pneumonia, endocarditis, and urogenital infections [5,6]. Likewise, our patient who hails from a TB endemic zone also presented with history and clinical presentation more suggestive of TB. However, a high degree of clinical awareness with timely and appropriate microbiological evaluation helped in an early definitive diagnosis. Moreover, it helped to avoid inappropriate administration of antitubercular medication on the patient.

The recommended management for splenic abscess has always been a combination of total splenectomy and appropriate antibiotic therapy. Few recent studies have proven that medical treatment and abscess drainage are equally effective [7-10]. However, various patient conditions contributing to the prognosis including the abscess number and size, underlying diseases, organism spectra, and general conditions are also to be taken into consideration. Our patient was young, immunocompetent, otherwise healthy, and with no other comorbidities. The splenic abscess was small, discrete, and solitary. $\mathrm{He}$, therefore, responded well to non-interventional approach. Limitation of the study being non-availability of follow-up scan due to the travel restrictions in association with the ongoing COVID-19 pandemic.

\section{CONCLUSION}

This case highlights that in this era of emerging infections, we should not miss the atypical presentations of the endemic diseases. Safe and minimally invasive radiological intervention with good microbiological correlation is a successful spleen conserving treatment alternative to surgery in suitable patients of splenic abscess.

\section{CONFLICTS OF INTEREST}

Nil.

\section{REFERENCES}

1. Alonso Cohen MA, Galera MJ, Ruiz M, Puig la Calle J, Ruis X, Artigas V, et al. Splenic abscess. World J Surg 1990;14:513-56.

2. Divyashree S, Gupta N. Splenic Abscess in immunocompetent patients managed primarily without splenectomy: A series of 7 cases. Perm J 2017;21:16-139.

3. Chang KC, Chuah SK, Changchien CS, Tsai TL, Lu SN, Chiu YC, et al. Clinical characteristics and prognostic factors of splenic abscess: A review of 67 cases in a single medical center of Taiwan. World $\mathrm{J}$ Gastroenterol 2006; 12:460-4

4. Kizilcan F, Tanyel FC, Büyükpamukçu N, Hiçsönmez A. Complications of typhoid fever requiring laparotomy during childhood. J Pediatr Surg 1993;28:1490-3.

5. Phillips GS, Radosevich MD, Lipsett PA. Splenic abscess: Another look at an old disease. Arch Surg 1997;132:1331-5.

6. Nasr B, Chhaider A, Mabrouk MB, Hamida MB, Farhat W, Othmen MB, et al. Diagnosis and treatment of splenic abscess (about 11 cases). J Gastroenterol Hepatol Res 2013;2:786-90.

7. Chiang IS, Lin TJ, Chiang IC, Tsai MS. Splenic abscesses: Review of 29 cases. Kaohsiung J Med Sce 2003;19:501-5.

8. Kumar S, Gupta V, Medappil N, Chandra A. Non-interventional management of splenic abscess. Ann Trop Med Public Health 2014;7:67-9.

9. Ferraioli G, Brunetti E, Gulizia R, Mariani G, MaroneP, Filice C. Management of splenic abscess: Report on 16 cases from a single center. Int J Infect Dis 2009;13:524-30.

10. Kattakayam AJ, Freeman MR, Karakattu SM, Johns SC, Youssef D, Pino P. A case for non-surgical management of splenic abscess in the setting of Salmonella typhimurium bacteremia. J Bacteriol Mycol Open Access 2019;7:64-6. 